\title{
Isolated huge right ventricular tumor: cardiac metastasis of tongue cancer
}

\author{
Sarah Chua, Wen-Hao Liu, and Wei-Chieh Lee
}

Division of Cardiology, Kaohsiung Chang Gung Memorial Hospital, Chang Gung University College of Medicine, Kaohsiung, Taiwan

Received: April 24, 2016

Revised : July 8, 2016

Accepted: September 28, 2016

\section{Correspondence to}

Wei-Chieh Lee, M.D.

Tel: +886-7-731-7123 (ext. 8300)

Fax: +886-7-732-2402

E-mail: leeweichieh@yahoo.com.tw
A 63-year-old male with a history of tongue cancer (5 years postresection and reconstruction) presented with progressive dyspnea during a 2-week period. Four months prior, a recurrence of the tongue cancer was diagnosed, for which he was undergoing concurrent chemoradiotherapy (CCRT). The parasternal long-axis view of transthoracic echocardiography (TTE) disclosed heterogeneous, multiple cavities, huge tumor of apical right ventricle (RV), with a minor pericardial effusion (Fig. 1A). The huge mass seemed like cheese with multiple central necrotic cavities (Fig. 1B). The short-axis view of TTE showed a D-shaped left ventricle (Fig. ${ }_{1 C}$ ). A normal pressure gradient was evident, with mild tricuspid regurgitation, and poor-to-borderline RV performance was noted. The reason of decreased RV performance may be related to tumor infiltrated to myocar- dium. The four-chamber view of TTE confirmed a bulky mass with adhering to apical and lateral aspects of RV and presented multiple necrotic cavities, based on focal hypodensity (Fig. 1D). Inferior vena cava and right atrium did not present any mass. Chest computed tomography showed a massive and centrally necrotic tumor of RV (Fig. 2A-2C), and did not detect obvious liver and lung metastases (Fig. $2 \mathrm{C}$ and 2D). Cardiac metastases secondary to squamous cell carcinoma of the tongue were strongly suspected. Adequate hydration and standard anti-heart failure treatment were performed for rightside heart failure. Then, the patient kept CCRT and shortness of breath improved gradually. The prevalence of oral cancers is high in Asian countries, especially in South and Southeast Asia. However, it is highly unusual for squamous cell carcinoma of the tongue to
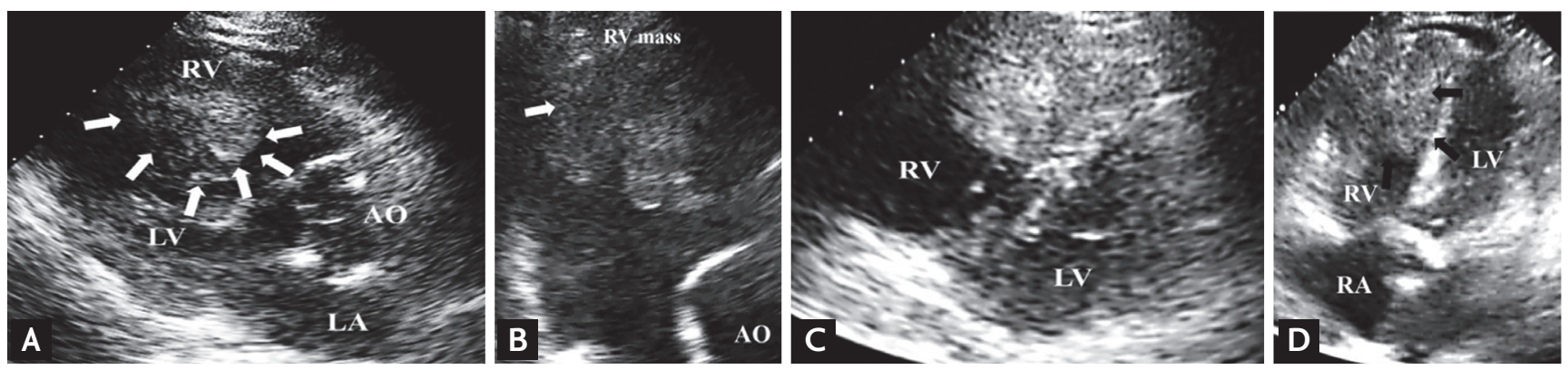

Figure 1. (A) Parasternal long-axis view of transthoracic echocardiography (TTE): huge heterogeneous mass in right ventricle (RV) (white arrows) and minor pericardial effusion. (B) Parasternal short-axis view of TTE: huge mass with multiple necrotic cavities (white arrow) and cheese-like picture. (C) Huge mass in RV and D-shaped left ventricle (LV). (D) Four-chamber view of TTE: the bulky mass of apical RV with multiple necrotic cavities (black arrows). AO, aorta. 

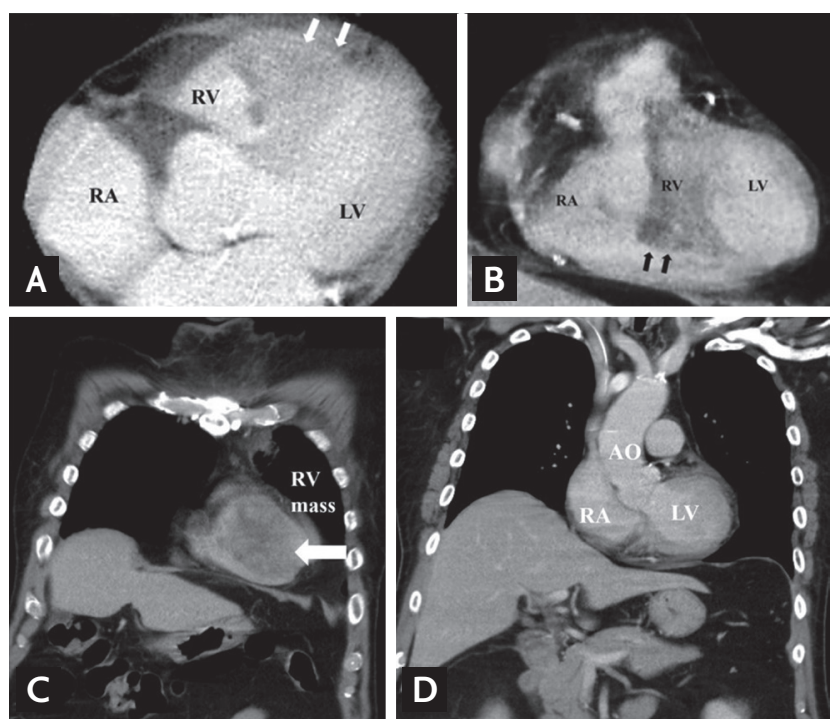

Figures 2. (A, B) Chest computed tomography: massive heterogeneous tumor of right ventricle (RV) with multiple necrotic cavities (white arrows in panel A, and black arrows in panel B). (C, D) Giant RV mass (white arrow) without obvious lung and liver metastases. $\mathrm{RA}$, right atrium; $\mathrm{LV}$, left ventricle; $\mathrm{AO}$, aorta. involve the heart. Due to rapid growth, cardiac metastasis of oral squamous cell carcinoma may develop central necrosis. Reported herein is an interesting and rare case of squamous cell carcinoma of the tongue with isolated RV metastases.

\section{Conflict of interest}

No potential conflict of interest relevant to this article was reported. 